\title{
Planar Quasi-Yagi Antenna Gain Enhancement Using Zero-Index Metamaterials
}

\author{
Roohollah Haghpanahan \\ School of Engineering and Design \\ Brunel University \\ London, UK \\ Roohollah.Haghpanahan@brunel.ac.uk
}

\author{
Rajagopal Nilavalan \\ School of Engineering and Design \\ Brunel University \\ London, UK
}

\begin{abstract}
In this paper we have applied a new anisotropic zero index metamaterial (ZIM) structure to the quasi-Yagi antenna to achieve a high-directivity quasi-Yagi antenna. Simulation results show the antenna gain increases over the bandwidth of anisotropic ZIM (10.3-11.7 GHz).
\end{abstract}

Keywords-Metamaterials, Quasi-Yagi antennas, Zero Index Material, Gain enhancement

\section{INTRODUCTION}

There has been a great interest in metamaterials in the past decade and plenty of metamaterial applications such as invisibility cloaks, negative refraction and zero index materials have been demonstrated. The anisotropic zero index materials -which exhibit zero permittivity or permeability in only one direction- show high efficiency to radiate and received electromagnetic waves and have been used to improve the antenna gain.

Planar antennas such as patch and slot antennas with wideband characteristics are effective for a portable terminal and have been the pursuit of antenna designers for many years. Microstrip patch antennas are inherently narrowband; although wider bandwidth could be achieved using techniques such as aperture coupling or introducing parasitic slot inside the patch, slot antenna with microstrip feeding but this will require more design and fabrication considerations. Temporarily, end-fire antennas such as Vivaldi present better bandwidth compare to patches or slots; however, they are usually larger than resonant patches or slots.

Planar quasi-Yagi antenna has both broadband characteristic of traveling wave radiators and the compactness of resonant type antenna and is useful in miniaturized communication systems because of its advantages such as low profile, low cost, lightweight, etc.

In this paper we have applied anisotropic ZIM to quasi-Yagi antenna to achieve a high-directivity quasi-Yagi antenna. The anisotropic ZIM structure can be well added to original quasiYagi antenna while proposed structure is still easy to fabricate.

\section{ANTENNA DESIGN AND RESULTS}

\section{A. Unit Cell Design}

A metamaterial structure is made of periodic unit cells which could be either resonant or non-resonant. In our design we have chosen a structure which is electrically resonant.

The frequency at which the permittivity is equal to zero is called plasma frequency. There are frequencies around the plasma frequency over which the permittivity is infinitesimal and consequently, the refractive index values are near zero. These frequencies define the bandwidth of a ZIM structure.

$$
n=\sqrt{\varepsilon \mu} \stackrel{|\varepsilon|<\delta}{\longrightarrow} 0
$$

Fig. 1 shows the unit cell structure was used in our design. The cell dimensions along both $\mathrm{x}$ - and $\mathrm{y}$-axis are $4 \mathrm{~mm}$. By treating the unit cell of the periodic structure as a particle, we obtained the effective parameters for a unit cell using standard retrieval method. As it is shown in Fig.1 when the electrical polarization is along $y$ - axis, an electrical resonance exists. The effective permittivity in both directions is shown in Fig.1. At frequencies around $11 \mathrm{GHz}$, the zero permittivity happens for waves travelling along $\mathrm{x}$-direction, while the permittivity along $y$-direction and permeability along $\mathrm{z}$-direction are constant. This will allow us to match the medium impedance to the air impedance for waves travelling along y-direction.
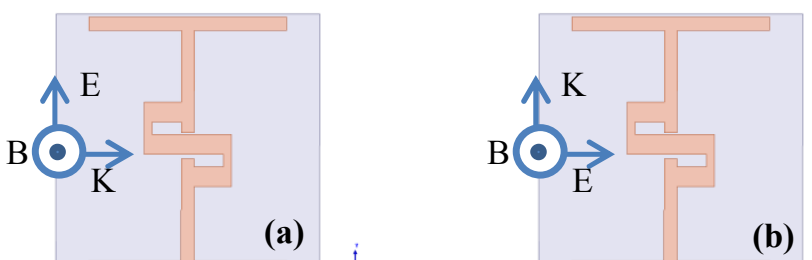


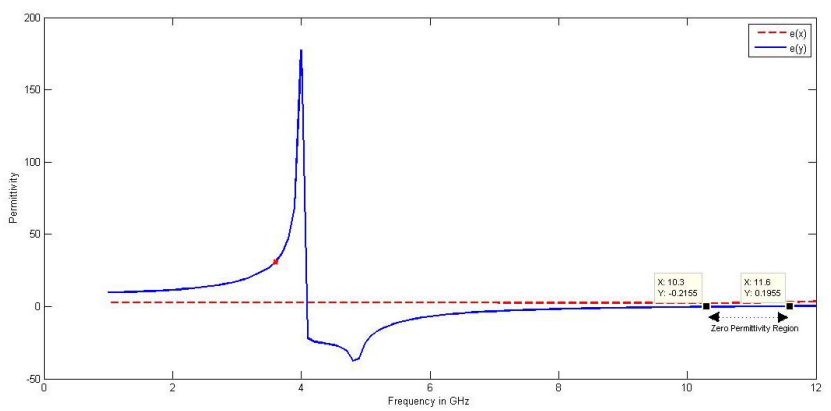

Fig.1. Designed unit cell with zero permittivity along $\mathrm{x}$-direction

\section{B. Proposed Antenna Design}

As it is demonstrated in Fig.2, the proposed ZIM structure was added to the original quasi-Yagi antenna. Designed antennas were simulated for different frequencies over the proposed ZIM bandwidth and results are shown in Fig2.b and c.
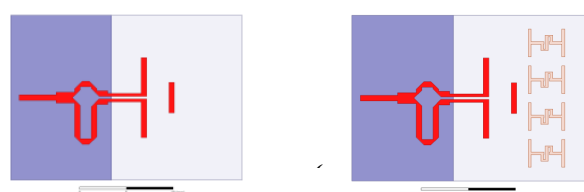

(a)

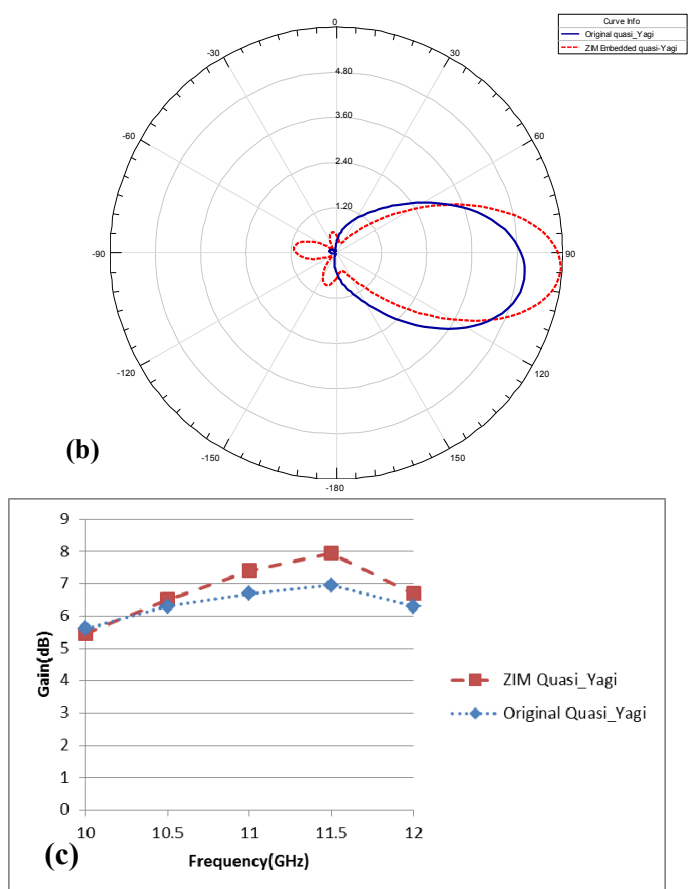

Fig2. (a) Original and ZIM embedded quasi-Yagi antenna design, (b) Antennas gain at $11.5 \mathrm{GHz}$, (c) Gain over ZIM bandwidth for original and ZIM enhanced quasi-Yagi antenna
The radiation pattern shows some reflections in the back of antenna due to slightly mismatch between the air impedance and the ZIM medium impedance. . Even better gain can be achieved by miniaturizing the backward reflection. This can be done by using optimization techniques. Further, we will apply this approach to array of quasi-Yagi antennas to obtain very high directivity antenna.

\section{CONCLUSION}

A novel planar ZIM structure was integrated to quasi-Yagi antenna. Gain enhancement achieved over the bandwidth of ZIM structure. The design is very simple and easy to fabricate. 